\title{
Óbito
}

\section{ERNEST ANTHONY LINER (1925-2010)}

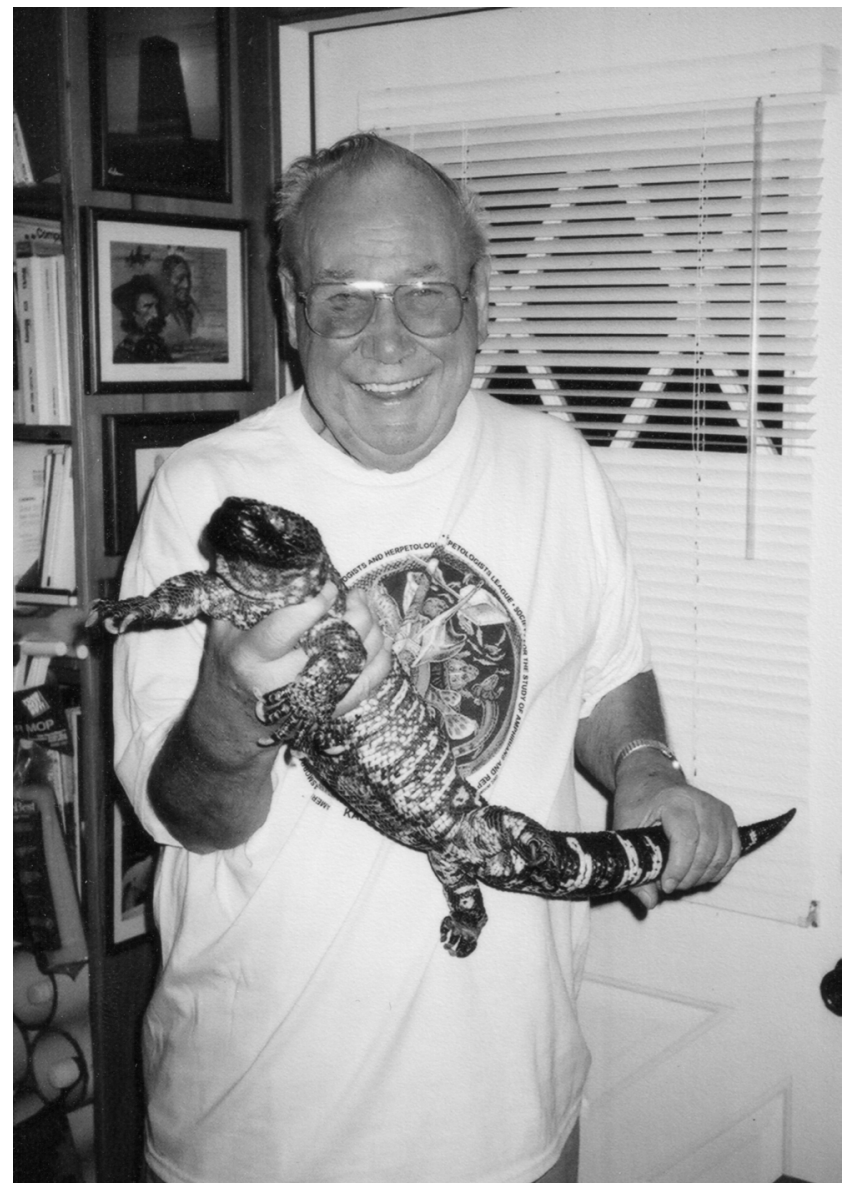

El Dr. Ernst A. Liner murió el día 23 de septiembre de 2010 en la ciudad de Houma, Louisiana en los Estados Unidos de América, lugar en que residía. Ernie -como se le conocía en el medio- tenía una edad de 85 años. Había nacido en el año de 1925 en la isla de Weeks, también en Louisiana.

Por cuanto a su vida familiar, Ernie tuvo dos hermanas, Mary Ann Fayette que lo precedió a su muerte y le sobreviven su otra hermana Jannette Liner Gomez y doce sobrinos. Durante la Segunda Guerra Mundial se enlistó en la Marina de los Estados Unidos y combatió para su país. Posteriormente, en 1955 se contrató con los laboratorios Squibb and Sons, donde trabajó por más de 32 años.

Recibido: 07 febrero 2011; aceptado: 03 marzo 2011
Ernest Liner se inició en la herpetología como un muy destacado aficionado y terminó convertido en un conocedor herpetólogo de tiempo completo, y más adelante obtuvo un grado de B.S. en Biología de la Louisiana State University. $\mathrm{Su}$ trabajo herpetológico lo realizaba en su casa o donde tuviera oportunidad de concentrarse.

Dentro de esta disciplina, su principal área de interés fue la herpetología de México y dedicó mucho de su tiempo a viajar a lo largo y ancho de este país. Su primer viaje a México, lo realizó en 1951 al estado de Nuevo León.

En 1952, Fred R. Cagle -uno de sus mentoreslo contrató para el Departamento de Zoología de Tulane University, donde se hizo cargo de la colección herpetológica durante tres años.

Dedicado a la labor herpetológica desde la década de 1940, el doctor Liner fue miembro activo de varias 
sociedades herpetológicas, dentro de las cuales publicó varios artículos que han sido muy apreciados por la comunidad internacional de herpetólogos. Durante su vida recibió muchos reconocimientos y homenajes. Uno de los más importantes fue el del doctorado honoris causa que le otorgó la Universidad de Colorado en el año de 1998.

Con gran carisma, Ernie fue muy apreciado por la comunidad, siempre mostraba una agradable y paciente sonrisa, era muy amable y una vez entablada la amistad con él, era amigo para toda la vida. Los que lo conocimos, tratamos y apreciamos, sin lugar a dudas que lo vamos a extrañar.

En cuanto a su producción científica, E. A. Liner publicó más de 130 artículos en diferentes revistas, la gran mayoría sobre herpetología, particularmente del sureste de Texas y noreste de México. Del total de artículos, más de 60 son sobre sistemática, distribución, historia natural y ecología de los anfibios y reptiles de México. También publicó más de 10 bibliografías e índices de artículos herpetológicos de diferentes revistas y realizó una compilación sobre los ejemplares tipo descritos por Hobart M. Smith. En colaboración con James R. Dixon, en el año 2003 publicaron una lista de los artículos herpetológicos que aparecen en los Anales del Instituto de Biología de la UNAM de 1930 a 1998 (vols.1-69).

Ernie describió varias especies y subespecies, entre las que se encuentran Pliocercus elapoides hobartsmithi (Liner, 1960), Tropidodipsas zweifeli (Liner y Wilson, 1970) y Sceloporus chaneyi (Liner y Dixon, 1992). Asimismo, se le dedicaron varias especies y subespecies de anfibios y reptiles como Cemophora coccinea lineri (Williams et al., 1966), Lepidophyma flavimaculatum lineri (Smith, 1973) y Thamnophis lineri (Rossman, 2005), y de otras especies de animales.

Los ejemplares recolectados por él se encuentran en museos, como el American Museum of Natural History, Louisiana State University, University of Kansas, entre otros. Ernie dispuso que su gran colección de libros y sobretiros de publicaciones se donaran a la American Society of Ichthyologist and Herpetologists, Herpetologists League y Society for the Study of Amphibians and Reptiles para que fueran subastados a su muerte.
Cabe destacar que las obras de síntesis más importantes que realizó sobre México en los últimos tiempos, fueron Checklist of the amphibians and reptiles of Mexico y la segunda edición de Nombres estándar en español y en inglés y nombres cientificos de los anfibios y reptiles de México (edición bilingüe), este último en colaboración con quien esto escribe. Varios de los artículos que publicó, fueron en colaboración con colegas mexicanos.

Además de sus contribuciones científicas, a Ernie mucho se le recordará por su gran interés y habilidad en cocinar, lo que puede apreciarse en su libro Culinary herpetology, publicado en el año de 2005.

Que descanse en paz Ernie.

Deseo agradecer al Dr. Joseph C. Mitchell, de la Mitchell Ecological Research Services, Gainesville, Florida por haberme facilitado la foto que aparece en este óbito.

Gustavo Casas Andreu Laboratorio de Herpetología Instituto de Biología,UNAM

\section{Bibliografía seleccionada para esta nota}

Dixon, J. R.y E. A. Liner. 2003. Herpetological papers published in Anales del Instituto de Biologia de la Universidad Nacional Autónoma de México. Boletín de la Sociedad Herpetológica Mexicana 11:57-74.

Liner, E. A. 2005. Culinary herpetology. Bibliomania, Salt Lake City, Utah. 392 p.

Liner, E. A. 2007. A checklist of the amphibians and reptiles of México. Occasional Papers of the Museum of Natural Science No. 80. Louisiana State University, Baton Rouge, Louisiana. $60 \mathrm{p}$.

Liner, E. A. y G. Casas Andreu. 2008. Nombres estándar en español y en inglés y nombres científicos de los anfibios y los reptiles de México - Standard Spanish, English and scientific names of the amphibians and reptiles of Mexico. Second edition. Society for the Study of Amphibians and Reptiles, Herpetological Circular No. 38. 162 p.

Mitchell, J. C. 2006. Ernest Anthony Liner. Copeia 2006:316320. 